\title{
Directional impulse response of a large cavity inside a sonic crystal
}

\author{
Ignacio Spiousas and Manuel C. Eguia ${ }^{\text {a) }}$ \\ Laboratorio de Acústica y Percepción Sonora, Universidad Nacional de Quilmes, R. S. Peña 352, Bernal, \\ B1876BXD Buenos Aires, Argentina
}

(Received 1 December 2011; revised 30 April 2012; accepted 18 June 2012)

\begin{abstract}
Both temporal and directional responses of a cavity inside a two-dimensional sonic crystal are investigated. The size of the cavity is large compared to the lattice parameter and the wavelength for the frequency range of interest. Hence, a hybrid method to compute the response is proposed, combining multiscattering theory for the calculation of the reflective properties of the sonic crystal with a modified ray-tracing algorithm for the sound propagation within the cavity. The response of this enclosure displays resonances for certain frequency bands that depend on the geometry of the lattice and the cavity. When a full band gap exists in the sonic crystal, rays cannot propagate through the medium and total reflection occurs for all incidence angles, leading to strong resonances with an isotropic intensity field inside the cavity. When only some propagation directions are forbidden, total reflection occurs for certain ranges of incidence angles, and resonances can also be elicited but with a highly anisotropic intensity field. The spectrum of resonances of the cavity is strongly affected by changes in the lattice geometry, suggesting that they can be tailored to some extent, a feature that can lead to potential applications in architectural acoustics.
\end{abstract}

(C) 2012 Acoustical Society of America. [http://dx.doi.org/10.1121/1.4744946]

PACS number(s): 43.55.Br, 43.20.Dk, 43.55.Ka, 43.20.Fn [ANN]

Pages: 2842-2851

\section{INTRODUCTION}

A two-dimensional sonic crystal is the simplest realization of an acoustic metamaterial, consisting of an array of rigid cylinders in air, that displays a large variation of its acoustical properties by just changing its geometrical configuration. For the past decade, sonic crystals (SCs) have been extensively studied due to their singular transmission properties (acoustic band gaps, ${ }^{1}$ negative refraction, ${ }^{2}$ negative bi-refraction, ${ }^{3}$ and focusing ${ }^{4}$ ). Equally singular, though less studied, ${ }^{5}$ are the reflective properties of SCs, and often with the only aim of enhancing the transmission properties (for example, designing anti-reflective acoustic coatings ${ }^{6}$ ). Most of the previous research also was devoted to the stationary sound field and only a few works studied the time dependent behavior. ${ }^{7}$

In this work we will focus on the reflective properties of SCs for a particular geometrical configuration: a large rectangular cavity inside a sonic crystal. We are interested in the temporal and angular properties of the sound field inside this enclosure and how they depend on the particular geometrical configuration of the SC. If the typical length of the cavity is much larger than the lattice constant we can think of this enclosure as analogous to a two dimensional room, with the walls replaced by sonic crystals. In contrast to what happens in ordinary rooms, where the acoustical properties of the walls are fixed, in this case the reflective properties of the sonic crystal walls can be continuously modified by changing their geometry, and consequently varying the acoustical properties of the sound field of the enclosure.

\footnotetext{
a) Author to whom correspondence should be addressed. Electronic mail: meguia@unq.edu.ar
}

The magnitude that completely characterizes sound field is the spatial impulse response, which corresponds to the sound pressure and particle velocity vector as a function of time and position inside the room, after being initially excited by an ideal pulse. ${ }^{8}$ In this article, we are interested in obtaining a slightly less informative quantity: the intensity vector as a function of time for a certain position of the room, which we termed the Energetic Directional Impulse Response (EDIR).

Several methods have been proposed so far to calculate the scattered field of SCs, including the plane wave expansion (PWE) method, ${ }^{9}$ multiple scattering theory (MST), ${ }^{10}$ variational methods, ${ }^{1}$ and finite difference time domain (FDTD) methods. ${ }^{11}$ In theory, all of them can be used to deal with SCs, but there are advantages and disadvantages to each method. In order to account for the EDIR inside a large cavity surrounded by SC walls, all these methods are either impractical or computationally infeasible. Hence, we propose an hybrid method, combining the calculation of the reflective properties of the SC walls using standard methods, with a geometrical acoustic approach for the sound field inside the cavity (which is valid as long as the cavity is large compared with the sound wavelength). ${ }^{12}$ In this way, we incorporate the advantages of the two methods. For the calculation of the reflective properties we use MST, which allows the fast computation of the field scattered from the walls using semianalytical calculations. While, for the sound field inside the cavity, we employ a modified ray-tracing method, incorporating diffraction.

This paper is organized as follows. We present the hybrid method for obtaining the impulse response in Sec. II. Numerical results for these quantities for different geometries are given in Sec. III. The Sec. IV is devoted to the 
discussion of the results, and a brief summary concludes the paper in the last section.

\section{METHODS}

In order to obtain the Energetic Directional Impulse Response (EDIR) of the cavity, we propose a novel hybrid method that combines multiscattering theory for the reflective properties of the walls and a ray tracing calculation for the cavity. The MST is the most used method to solve the scattered field of acoustic periodic structures and the ray tracing method is a standard procedure for the calculation of the Impulse Response in room acoustics. The hybrid method was developed in order to reduce the computation time compared to brute force time domain wave based methods (like FDTD), making the study of parametric variations of the cavity geometry and reflective properties computationally feasible.

\section{A. Multiple scattering theory}

We begin by deriving the reflectivity index and the scattered angle probability for an infinite sonic crystal slab. This will allow us to use these magnitudes as an input for the ray tracing method in the next section; due to the fact that in the context of geometric acoustics, we can consider each ray as a portion of a plane wave impinging an infinite wall.

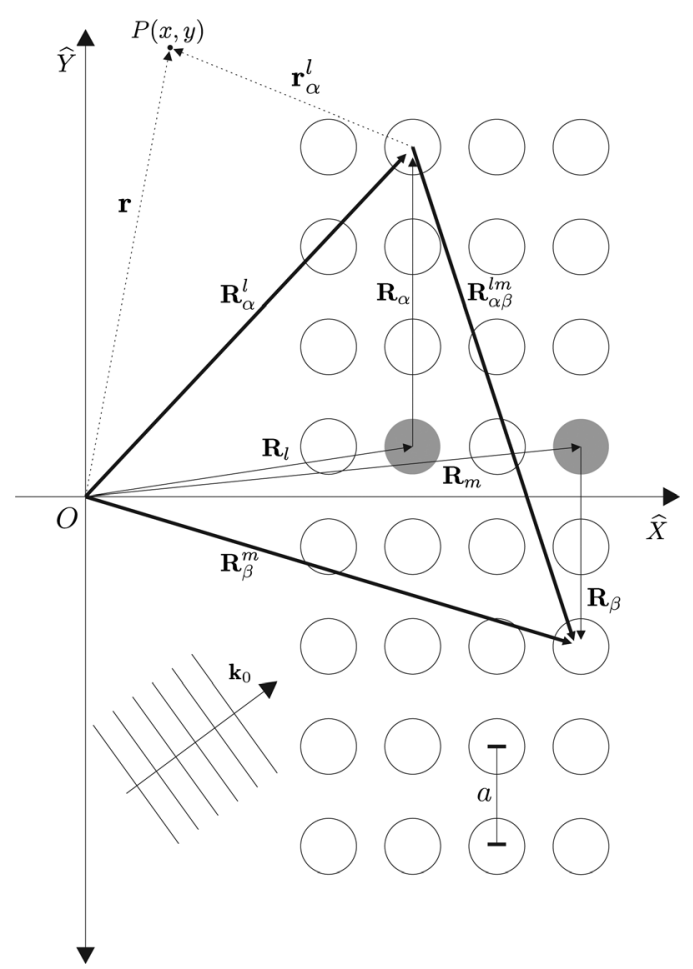

FIG. 1. Geometric definitions for the infinite rows of cylinders (Sonic Crystal walls) with lattice parameter $a$, used for multiple scattering theory calculations. The position vector for the $\alpha_{t h}$ cylinder of the $l_{t h}$ row is defined by $\mathbf{R}_{\alpha}^{l}=\mathbf{R}_{l}+\mathbf{R}_{\alpha}$, where $\mathbf{R}_{l}=\left(x_{l}, y_{l}\right)$ sets the position of the central cylinder in a row while the vector $\mathbf{R}_{\alpha}=(0, \alpha a)$ determines the position of the $\alpha_{\text {th }}$ cylinder relative to the central one. The distance vector between the $(\alpha, l)$ cylinder and the $(\beta, m)$-cylinder is determined by $\mathbf{R}_{\alpha \beta}^{l m}=\mathbf{R}_{\beta}^{m}-\mathbf{R}_{\alpha}^{l}$. The position vector of the evaluation point $P(x, y)$ relative to the $(\alpha, l)$-cylinder is defined by $\mathbf{r}_{\alpha}^{l}=\mathbf{r}-\mathbf{R}_{\alpha}^{l}$ where $r$ stands for its position relative to the origin. $\mathbf{k}_{0}$ represents the wave vector of the incident field.
In Fig. 1 we depict a portion of the SC wall for the case of a square lattice. Rows of cylinders are infinite in the $\hat{\mathbf{Y}}$ direction, and the incident field is a plane wave with wave vector $\mathbf{k}_{0}=k\left(\cos \theta_{0}, \sin \theta_{0}\right)$ forming an angle $\theta_{0} \in[-\pi / 2, \pi / 2]$ with the positive $\hat{\mathbf{X}}$ axis (normal to the wall). Unitary vectors in capital letters are used for axes relatives to the wall, in order to distinguish them from the axes for the cavity. The lattice parameter is denoted by $a$ and the cylinder radius by $\rho$.

The position vector for the $\alpha_{t h}$ cylinder of the $l_{t h}$ row is defined by

$$
\mathbf{R}_{\alpha}^{l}=\mathbf{R}_{l}+\mathbf{R}_{\alpha}=x_{l} \hat{\mathbf{x}}+\left(y_{l}+\alpha a\right) \hat{\mathbf{y}}
$$

where $\alpha$ goes from $-\infty$ to $\infty$. The vector $\mathbf{R}_{l}=\left(x_{l}, y_{l}\right)$ defines the position of the central cylinder in a row, while the vector $\mathbf{R}_{\alpha}=(0, \alpha a)$ determines the position of the $\alpha_{t h}$ cylinder relative to the central one. In Fig. 1 we also display the position vector $\mathbf{R}_{\beta}^{m}$ for the $\beta_{t h}$ cylinder of the $m_{t h}$ row, the difference vector $\mathbf{R}_{\alpha \beta}^{l m}=\mathbf{R}_{\beta}^{m}-\mathbf{R}_{\alpha}^{l}$, and the position vector of the evaluation point $r$ relative to the origin and relative to the $\alpha_{t h}$ cylinder of the $l_{t h}$ row $\mathbf{r}_{\alpha}^{l}$. The angles corresponding to these position vectors are denoted by the greek letter $\theta$ with the same sub and superindices. For example, $\theta_{\alpha}^{l}$ corresponds to the angle formed by $\mathbf{R}_{\alpha}^{l}$ with the positive $\hat{\mathbf{X}}$ axis.

We now turn on the MST formulation of the problem. This theory solves the problem of multiple scattering of cylinders by taking the exact solution of a single scatterer and extending it to an array by means of the Graft's addition theorem that allows a coordinate change to the scatterer's coordinate system. ${ }^{13}$

For the case of the infinite SC wall the scatterers are indexed by the row number $l$ and the cylinder order in the row $\alpha$. The total incident field on the $(\alpha, l)$-cylinder in the scatterer's coordinate system $P_{\alpha l}^{0}\left(\mathbf{r}_{\alpha}^{l}\right)$ can be expressed in terms of Bessel functions of the first kind:

$$
P_{\alpha l}^{0}\left(\mathbf{r}_{\alpha}^{l}\right)=\sum_{q}\left(B_{\alpha}^{l}\right)_{q} J_{q}\left(k r_{\alpha}^{l}\right) e^{i q \theta_{\alpha}^{l}}
$$

and the scattered field of the same cylinder in the central coordinate system $P_{\alpha l}^{S C}(\mathbf{r})$ can be expanded in terms of Hankel functions of the first kind:

$$
P_{\alpha l}^{S C}(\mathbf{r})=\sum_{q}\left(A_{\alpha}^{l}\right)_{q} H_{q}\left(k r_{\alpha}^{l}\right) e^{i q \theta_{\alpha}^{l}} .
$$

The coefficients of the expansions of the incident and scattered fields are related by means of the T-matrix of the cylinder:

$$
\left(A_{\alpha}^{l}\right)_{q}=T_{q}\left(B_{\alpha}^{l}\right)_{q}
$$

that for the case of perfectly rigid cylinder is ${ }^{14} T_{q}$ $=-J_{q}^{\prime}(k \rho) / H_{q}^{\prime}(k \rho)$.

Using the Graft's addition theorem, the total incident field on the $(\alpha, l)$-cylinder can also be expressed as a sum of the external incident field and the scattered field from the other cylinders: 


$$
\begin{aligned}
& P_{\alpha l}^{0}\left(\mathbf{r}_{\alpha}^{l}\right)=P^{e x t}\left(\mathbf{r}_{\alpha}^{l}\right)+\sum_{(\beta, m) \neq(\alpha, l)} P_{\beta m}^{S C}\left(\mathbf{r}_{\alpha}^{l}\right), \\
& P^{e x t}\left(\mathbf{r}_{\alpha}^{l}\right)=\sum_{q} A_{q}^{0} e^{i \mathbf{k}_{0} \cdot \mathbf{R}_{\alpha}^{l}} J_{q}\left(k r_{\alpha}^{l}\right) e^{i q \theta_{\alpha}^{l}}, \\
& P_{\beta m}^{S C}\left(\mathbf{r}_{\alpha}^{l}\right)=\sum_{q}\left(A_{\beta}^{m}\right)_{s} H_{q-S}\left(k R_{\alpha \beta}^{l m}\right) e^{i(s-q) \theta_{\alpha \beta}^{l m}} J_{q}\left(k r_{\alpha}^{l}\right) e^{i q \theta_{\alpha}^{l}},
\end{aligned}
$$

where $A_{q}^{0}=i^{q} e^{-i q \theta_{0}}$ are the Bessel expansion coefficients of the external incident plane wave. The summation on the right hand side of the Eq. (5) is over a finite number of rows indexed by $m$, and over $\beta$ going from $-\infty$ to $\infty$ (with the exception of $(\beta, m)=(\alpha, l))$.

After multiplying Eqs. (2) and (5)-(7) by $T_{q}$, using Eq. (4) and equating the coefficients of the expansions, the MST equation for the infinite SC wall is obtained ${ }^{15,16}$ :

$$
\left(A_{\alpha}^{l}\right)_{q}-\sum_{s} \sum_{m} \sum_{\beta}\left(G_{\alpha \beta}^{l m}\right)_{q s}\left(A_{\beta}^{m}\right)_{s}=T_{q} A_{q}^{0} e^{i \mathbf{k}_{0} \cdot \mathbf{R}_{l}} e^{i \mathbf{k}_{0} \cdot \mathbf{R}_{\alpha}},
$$

where $\left(G_{\alpha \beta}^{l m}\right)_{q s}$, the propagator from $(\beta, \mathrm{m})$ to $(\alpha, 1)$ is defined by

$$
\left(G_{\alpha \beta}^{l m}\right)_{q s}=\left(1-\delta_{\alpha \beta} \delta_{l m}\right) T_{q} H_{q-s}\left(k R_{\alpha \beta}^{l m}\right) e^{i(s-q) \theta_{\alpha \beta}^{l m}} .
$$

As the external field is a plane wave, the problem becomes symmetrical relative to a translation along the $\hat{\mathbf{Y}}$ direction, then $\left(G_{\alpha \beta}^{l m}\right)_{q s}=\left(G_{0 \beta-\alpha}^{l m}\right)_{q s}$ and the scattering from the $\alpha_{t h}$ cylinder of the $l_{t h}$ row differs from the scattering of the central cylinder of that row only by a phase factor: $e^{i \mathbf{k}_{0} \cdot \mathbf{R}_{\alpha}}=e^{i \alpha k a \sin \theta_{0}}$. We can get rid of this phase factor by multiplying both sides of Eq. (8) by $e^{-i \alpha k a \sin \theta_{0}}$, writing down each term of the multiple sum as:

$$
e^{-i \alpha k a \sin \theta_{0}}\left(G_{\alpha \beta}^{l m}\right)_{q s}\left(A_{\beta}^{m}\right)_{s}=\left(G_{\alpha \beta}^{l m}\right)_{q s} e^{i(\beta-\alpha) k a \sin \theta_{0}} e^{-i \beta k a \sin \theta_{0}}\left(A_{\beta}^{m}\right)_{s}
$$

and changing the summation index to $\beta-\alpha$. Then, new terms independent of $\alpha$ can be defined as

$$
\begin{aligned}
& \left(A_{l}\right)_{q}=e^{-i \alpha k a \sin \theta_{0}}\left(A_{\alpha}^{l}\right)_{q}, \\
& \left(g_{l m}\right)_{q s}=\sum_{\beta=-\infty}^{\infty}\left(G_{0 \beta}^{l m}\right)_{q s} e^{i \beta k a \sin \theta_{0}},
\end{aligned}
$$

and Eq. (8) can be rewritten as

$$
\left(A_{l}\right)_{q}-\sum_{s} \sum_{m}\left(g_{l m}\right)_{q s}\left(A_{m}\right)_{s}=T_{q} e^{\mathbf{k}_{0} \cdot \mathbf{R}_{l}} i^{q} e^{-i q \theta_{0}} .
$$

Now the MST equation is expressed in terms of scattering of infinite rows, instead of cylinders. The propagator matrix $\left(g_{l m}\right)_{q s}$ can be calculated using Eqs. (12) and (9), but in order to improve the convergence of the calculation, the infinite sum in $\beta$ can be alternatively expressed as summation over the complex diffraction orders of the rows. ${ }^{16}$

We consider first the case when $l \neq m$ and the propagator between rows can be rewritten as

$$
\left(g_{l m}\right)_{q s}=T_{q}(-1)^{q-s} \sum_{\beta=-\infty}^{\infty} H_{s-q}\left(k R_{0 \beta}^{l m}\right) e^{i(s-q) \theta_{0 \beta}^{l m}} e^{i \beta k a \sin \theta_{0}}
$$

The lattice sum over $\beta$ can be performed starting from the following integral representation of the Hankel function:

$$
H_{s-q}\left(k R_{0 \beta}^{l m}\right) e^{i(s-q) \theta_{0 \beta}^{l m}}=\frac{i^{q-s}}{\pi} \int_{C} e^{i \mathbf{k}_{\theta} \cdot \mathbf{R}_{0 \beta}^{l m}} e^{i(s-q) \theta} d \theta,
$$

where $\mathbf{k}_{\theta}=k(\cos \theta, \sin \theta)$ and the integration path $\mathrm{C}$ is a line from $\theta_{i}=i \infty-\pi / 2(i \infty+\pi / 2)$ to $\theta_{f}=-i \infty+\pi / 2$ $(-i \infty+3 \pi / 2)$ if $m>l(m<l)$. The position vector can be rewritten $\mathbf{R}_{0 \beta}^{l m}=\mathbf{R}_{m}-\mathbf{R}_{l}-\beta a \hat{y}$, and the sum over $\beta$ can be commuted with the integral. Therefore, the propagator can be expressed as

$$
\begin{aligned}
\left(g_{l m}\right)_{q s}= & T_{q} \frac{(-i)^{q-s}}{\pi} \int_{C} e^{i \mathbf{k}_{\theta} \cdot\left(\mathbf{R}_{m}-\mathbf{R}_{l}\right)} e^{i(s-q) \theta} \\
& \times \sum_{\beta=-\infty}^{\infty} e^{i \beta k a \sin \left(\theta_{0}-\theta\right)} d \theta
\end{aligned}
$$

Now the infinite sum can be transformed to a Dirac comb:

$$
\sum_{\beta=-\infty}^{\infty} e^{i \beta k a \sin \left(\theta_{0}-\theta\right)}=\frac{2 \pi}{k a} \sum_{\nu=-\infty}^{\infty} \delta\left(\sin \theta_{0}-\sin \theta-\frac{2 \pi \nu}{k a}\right) .
$$

After making the change $t=\sin \theta$ and converting the integral over the path $C$ into an integral over the interval $(-\infty, \infty)$, the Dirac comb can be integrated yielding the following expression for the propagator ${ }^{16}$ :

$$
\left(g_{l m}\right)_{q s}=T_{q} \frac{2(-i)^{q-s}}{k a} \sum_{\nu} \frac{e^{i(q-s) \theta_{\nu}}}{\left|\cos \theta_{\nu}\right|} e^{i \mathbf{k}_{\nu}^{ \pm} \cdot\left(\mathbf{R}_{\mathbf{m}}-\mathbf{R}_{\mathbf{l}}\right)},
$$

where the subindex $\nu$ was added to the integrated values of $\mathbf{k}$ and $\theta$. The wave vector $\mathbf{k}_{\nu}$ and angle $\theta_{\nu}$ are defined from

$$
\begin{aligned}
& \sin \theta_{\nu}=\sin \theta_{0}-(2 \pi \nu) /(k a) \\
& \cos \theta_{\nu}= \pm \sqrt{1-\sin ^{2} \theta_{\nu}} \\
& \mathbf{k}_{\nu}^{ \pm}=k\left(\cos \theta_{\nu}, \sin \theta_{\nu}\right)
\end{aligned}
$$

where and a plus (minus) sign in the superscripts corresponds to the case $l>m(m>l)$.

For the case $l=m$ in Eq. (12), the lattice sum over $\beta$ has to be taken excluding the case $\beta=0$ and the formula of Eq. (17) cannot be applied. Since the position vector and 
angle are calculated within the same row, they take the following simple form: $R_{0 \beta}^{l l}=\beta a$ and $\theta_{0 \beta}^{l l}= \pm \pi / 2$, where the sign is (positive) for $\beta$ positive (negative). Then the diagonal term of the propagator can be rewritten as

$$
\begin{aligned}
\left(g_{l l}\right)_{q s}= & T_{q}(-1)^{q-s} \sum_{\beta \neq 0} H_{s-q}(\beta k a) i^{s-q}(-1)^{\beta /|\beta|} e^{i \beta k a \sin \theta_{0}} \\
= & T_{q}(-i)^{s-q} \sum_{\beta=1}^{\infty} H_{s-q}(\beta k a) \\
& \times\left[e^{i \beta k a \sin \theta_{0}}+(-1)^{s-q} e^{-i \beta k a \sin \theta_{0}}\right] .
\end{aligned}
$$

Applying the same integral form of Hankel function used in Eq. (15) and after some algebraic manipulation Eq. (22) becomes:

$$
\left(g_{l l}\right)_{q s}=T_{q}\left[S_{s-q}^{-}+(-1)^{s-q} S_{s-q}^{+}\right]
$$

where $S_{s-q}^{ \pm}$corresponds to the incomplete lattice sum:

$$
\begin{aligned}
S_{s-q}^{ \pm}= & \frac{e^{-i \pi / 4}}{\pi} e^{ \pm i k a \sin \theta_{0}} \\
& \times \int_{-\infty}^{\infty} \frac{e^{i k a \sqrt{1+i t^{2}}}\left[\sqrt{1+i t^{2}}+i t e^{-i \pi / 4}\right]^{s-q}}{\sqrt{1+i t^{2}}\left[1-e^{i k a \sqrt{1+i t^{2}}} e^{ \pm i k a \sin \theta_{0}}\right]} d t
\end{aligned}
$$

that can be computed numerically.

Now the values of $\left(g_{l m}\right)_{q s}$ can be calculated using Eqs. (18), (24), and (25) and truncating the summation over $\nu$, due to the good convergence of the complex diffraction modes. Also, the convergence of the series of Hankel function is good enough to allow the calculation of the scattering coefficients $\left(A_{l}\right)_{q}$ from Eq. (13) truncating the sum and using standard algebra.

Once the scattering coefficients for the rows $\left(A_{l}\right)_{q}$ are determined, the total scattered pressure field can be expressed as a sum of the scattered fields over all $(\alpha, l)$ cylinders given by Eq. (3), with the coefficients $\left(A_{\alpha}^{l}\right)_{q}$ obtained from Eq. (11):

$$
P^{S C}(\mathbf{r})=\sum_{q} \sum_{\alpha} \sum_{l}\left(A_{\alpha}^{l}\right)_{q} H_{q}\left(k r_{\alpha}^{l}\right) e^{i q \theta_{\alpha}^{l}}
$$

Again, the infinite sum over $\alpha$ can be replaced with a summation over the Bragg order numbers $\nu$, following the same derivation of Eqs. (15)-(18), ${ }^{16}$ and the total scattered field can be written down as

$$
P^{S C}(\mathbf{r})=\sum_{\nu} C_{\nu}^{ \pm} e^{i \mathbf{k}_{\nu}^{ \pm}} \cdot \mathbf{r}
$$

where now a plus (minus) sign correspond to $x>x_{l}\left(x<x_{l}\right)$ and $C_{\nu}^{ \pm}$is the amplitude of the $\nu_{t h}$ scattered mode:

$$
C_{\nu}^{ \pm}=\sum_{q} \sum_{l}\left(A_{l}\right)_{q} \frac{2(-i)^{-q}}{k a} \frac{e^{i q \theta_{\nu}}}{\left|\cos \theta_{\nu}\right|} e^{-i \mathbf{k}_{\nu}^{ \pm} \cdot \mathbf{R}_{l}} .
$$

In general $\mathbf{k}_{\nu}$ and $\theta_{\nu}$ are complex, but for $\nu$ belonging to the interval between $\nu_{-}=(k a / 2 \pi)\left(\sin \theta_{0}-1\right)$ and $\nu_{+}=$ $(k a / 2 \pi)\left(\sin \theta_{0}+1\right)$ those values are real and correspond to the wave vector and diffraction angle of the $\nu_{t h}$ Bragg order, respectively. Since in the following we will be only interested in the propagating reflected modes, we will take $\nu$ within the interval $\left[\nu_{-}, \nu_{+}\right]$and use the minus sign in Eq. (28).

From the amplitudes of the scattered modes, we can calculate the reflectivity matrix $(\mathcal{R})$, that represents the intensity for every reflected angle $\left(\alpha_{r}\right)$ for a given plane wave of angle of incidence $\left(\alpha_{i}\right)$ and frequency $(f)$.

$$
\mathcal{R}\left(f, \alpha_{i}, \alpha_{r}\right)=\left\{\begin{array}{ll}
\frac{\left|\cos \alpha_{r}\right|}{\cos \alpha_{i}}\left|C_{\nu}^{-}\left(f, \alpha_{i}\right)\right|^{2} & \text { if } \alpha_{r}=\theta_{\nu}\left(f, \alpha_{i}\right) \\
0 & \text { if } \alpha_{r} \neq \theta_{\nu}\left(f, \alpha_{i}\right)
\end{array},\right.
$$

where only the $C_{\nu}^{-}$are used for the reflective properties of the slab, and $\theta_{\nu}$ is given by Eq. (19) with $\theta_{0}=\alpha_{i}$. Also, we make explicit the dependence of these coefficients on the frequency of the incident ray $f$ and the incidence angle $\alpha_{i}$.

\section{B. Ray tracing method}

The Ray tracing method describes the sound propagation under the approximation of geometrical acoustics, using rays corresponding to small portions of plane waves of a certain frequency emanating from a source $(\Sigma)$. This approximation is valid as long as the wavelength is small compared with the typical length of the cavity. ${ }^{12}$ Rays propagate with constant energy and direction inside the cavity, and after hitting a wall they change their energy and direction according to the reflectivity properties of the boundary. They are traced around until the energy decay below some fixed value $E_{\min }$.

A number of acoustical parameters and the energetic impulse response can be calculated for any point inside the cavity from this calculation, provided that a sufficient number of rays (typically severals tens of thousands) are used.

Standard ray tracing methods in acoustics incorporate specular and diffuse reflection but no diffraction. In our method, we incorporate the Bragg diffraction at the walls using the results derived in the previous section.

Each ray segment (between reflections) is characterized by its direction, energy and the frequency of the associated wave. After a reflection, a new energy and direction is determined. The energy is multiplied by a factor equal to the total reflectivity index, that can be calculated integrating $\mathcal{R}$ over all reflected angles $\alpha_{r}$, or equivalently summing Eq. (29) over the possible reflection angles $\theta_{\nu}$. The new direction is selected among the possible Bragg orders $\nu$ using probabilities determined from the amplitudes $\left|C_{\nu}^{-}\right|$from Eq. (28). In order to make the computations of reflection faster, we precalculate two quantities that can be stored in matrix form: (a) the Reflectivity index $R\left(f, \alpha_{i}\right)$, and (b) the scattered angle probability density $S\left(f, \alpha_{i}, \alpha_{r}\right)$, which are defined as follows:

$$
R\left(f, \alpha_{i}\right)=\sum_{\theta_{\nu}} \frac{\left|\cos \theta_{\nu}\right|}{\cos \theta_{\nu}}\left|\mathbf{C}_{\nu}^{-}\left(f, \alpha_{i}\right)\right|^{2}
$$




$$
S\left(f, \alpha_{i}, \alpha_{r}\right)=\left\{\begin{array}{ll}
\frac{1}{\sum_{\alpha_{r}} \mathrm{C}_{\nu}^{-}\left(f, \alpha_{i}\right)} & \text { if } \alpha_{r}=\theta_{\nu}\left(f, \alpha_{i}\right) . \\
\text { if } \alpha_{r} \neq \theta_{\nu}\left(f, \alpha_{i}\right)
\end{array} .\right.
$$

Once all ray segments are calculated for a particular frequency $(f)$, an arbitrary position of the receiver $\Delta$ is selected, taking a finite size disk (detector) of radius $\delta$. All ray segments crossing the detector are extracted, storing their time of arrival $t_{n}(f)$, energy $E_{n}(f)$ and direction $\alpha_{n}(f)$. Hence, an energetic directional impulse response $(H)$ can be calculated for the chosen source/receiver pair:

$$
H_{\Sigma \Delta}(t, f, \alpha)=\sum_{n} \delta\left(t-t_{n}(f)\right) \delta\left(\alpha-\alpha_{n}(f)\right) E_{n}(f),
$$

note that in this equation the angle $\alpha$ runs from $-\pi$ to $\pi$ and is taken as the angle with the $\hat{x}$ direction. This energetic directional impulse response completely characterizes the temporal, spectral and angular behavior of the instantaneous intensity vector for the particular positions of the source and the receiver. For the sake of clarity, we derive from this response two different magnitudes: (a) the Remaining Energy Density RED, and (b) the Angular Energy Density AED; the first containing the temporal information and the second the directional information of $H$ as a function of frequency. The $\operatorname{RED}(f, t)$ corresponds to the energy remaining in the cavity at time $t$ for a particular frequency $f$ and it is obtained by integrating over all arrival angles $(\alpha)$, and integrating backwards in time (a method due to Schroeder ${ }^{17}$ ):

$$
R E D(f, t)=\int_{\tau=t}^{\infty} \int_{\alpha=-\pi}^{\pi} H(\tau, f, \alpha) d \alpha d \tau .
$$

The $A E D(f, t)$, in turn, corresponds to the intensity integrated for all times as a function of the arrival angle $\alpha$ and frequency $f$, and is calculated by direct time integration:

$$
A E D(f, \alpha)=\int_{t=0}^{\infty} H(t, f, \alpha) d t
$$

These two quantities will be studied for a rectangular cavity in the following section for different SC geometries.

\section{RESULTS}

In this section we present the results of the averaged EDIR quantities $[R E D(f, t)$ and $A E D(f, \alpha)]$ using the hybrid method developed before. Since we are interested on how the EDIR of the cavity can be modified by changing the lattice geometry of the sonic crystal walls, we explore three different configurations: (a) a square lattice sonic crystal, (b) a hexagonal lattice sonic crystal, and (c) a perturbed hexagonal lattice sonic crystal. For all three cases the SCs walls are eight infinite rows of rigid cylinders with radius $\rho=0.08 \mathrm{~m}$ embedded on air, and the rectangular cavity has the following arbitrary dimensions: $L_{x}=7.61 \mathrm{~m} \hat{x}$ and $L_{y}=4.63 \mathrm{~m} \hat{y}$. Since we will discuss the analogy with a real room, we keep the numerical values for the geometrical parameters and frequency, instead of using non-dimensional magnitudes.

\section{A. Square lattice}

For the first configuration to be analyzed, the walls of the cavity are square lattice sonic crystals with lattice parameter $a=0.1861 \mathrm{~m}$. As the cavity is rectangular and the square lattice is symmetric with respect to a right angle rotation, the scattering properties of the cavity are the same for all walls.

The first step of the hybrid method presented here is to perform the MST calculation in order to derive the scattering properties of the sonic crystal walls. A frequency range between 0.1 and $4 \mathrm{kHz}$, in steps of $20 \mathrm{~Hz}$ was explored. For this configuration, the position of the central cylinder for the line $l$ is given by $R_{l}=l a \hat{X}$ and the position of cylinder $\alpha$ of line $l$ by $R_{\alpha}=\alpha a \hat{Y}$ (Fig. 1).

In Fig. 2(a) we display the Reflectivity index matrix obtained using MST for the square lattice with the parameters given above. As a reference, in Fig. 2(b), we also display the acoustic band structure diagram in the first Brillouin zone of the lattice, calculated using the PWE method. For the reflectivity index matrix $R\left(f, \alpha_{i}\right)$ two features are apparent: the existence of regions with reflectivity equal to one (total reflection) and the presence of fringes next to these regions. The total reflection regions can occupy a full frequency band, for all possible incidence angles (as occurs between 0.865 and $1.25 \mathrm{kHz}$ ) or form patterns with typical "M" shapes, where total reflection happens only for certain a)

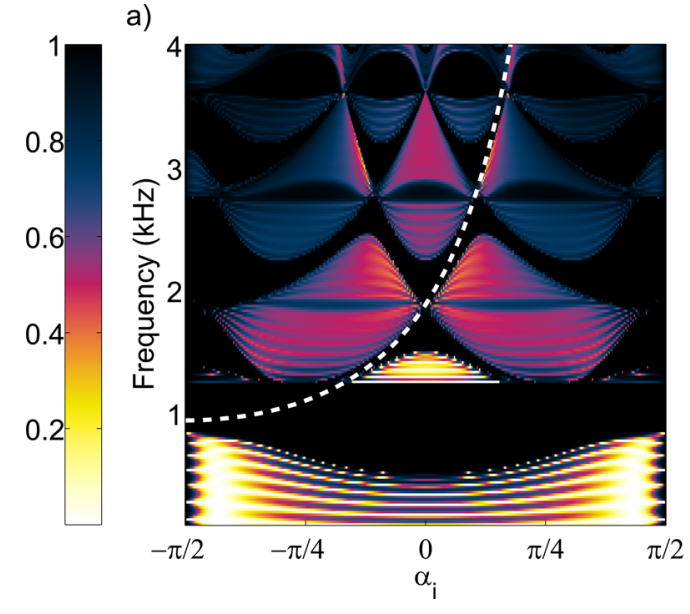

b)

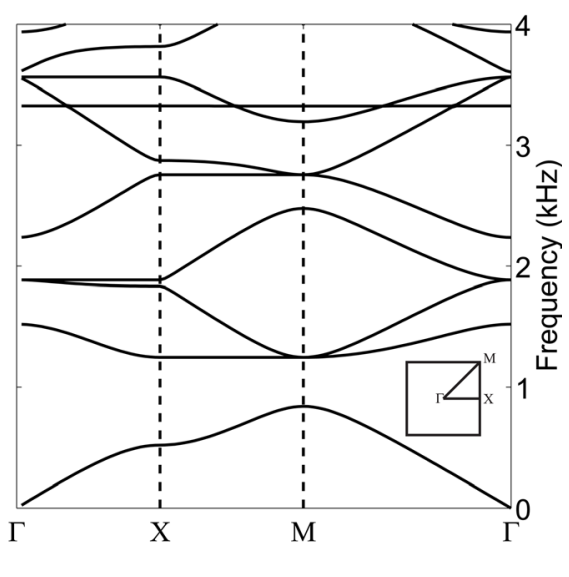

FIG. 2. (Color online) (a) Reflectivity index matrix, $R\left(f, \alpha_{i}\right)$, for a square lattice $(a=0.1816 \mathrm{~m}, \rho=0.08 \mathrm{~m})$ sonic crystal infinite slab obtained using MST. This matrix gives the reflectance coefficient for each frequency $(f)$ and angle of incidence $\left(\alpha_{i}\right)$. The superimposed dashed gray line corresponds to the beginning of one of the first Bragg orders given by $(k a / 2 \pi)\left(\sin \alpha_{i}-1\right)=-1$. (b) As a reference we display the band structure diagram for the same lattice calculated using PWE. The horizontal axis correspond to the path $\Gamma, X, M$, $\Gamma$ in the first Brillouin zone (inset). 
incidence angles. When comparing these regions with the band diagram, it arrives that the full reflection frequency regions correspond to full band gaps of the sonic crystal. The "M" shape patterns, in turn, correspond to regions where the sonic crystal has partial band gaps. As the fringes are related with Fabry-Perot Resonances the number of them is directly proportional to the number of rows of the sonic crystal.

The scattered angle probability density $S\left(f, \alpha_{i}, \alpha_{r}\right)$, is displayed in Fig. 3(a) for a particular value of frequency $f=2.5 \mathrm{kHz}$. This matrix represents, for a given ray with angle of incidence $\alpha_{i}$, the probability of being reflected at an angle $\alpha_{r}$. For an infinite SC slab the only possible values of $\alpha_{r}$ correspond to the Bragg angles, as displayed in Fig. 3(b).

The analysis of $S\left(f, \alpha_{i}, \alpha_{r}\right)$ is more complex, since a scattered angle probability matrix is obtained for each frequency. In Fig. 3 the matrix for $f=2.5 \mathrm{kHz}$ is displayed as an example. As it can be expected, the matrix has nonzero values only for the angles corresponding to the different Bragg orders, from $\nu=0$ corresponding to the anti-diagonal (specular reflection) to ascending orders corresponding to subsequent concentric arches. However, for a given angle of incidence, the probability of being diffracted to a certain Bragg order is far from trivial.

Once the quantities $R$ and $S$ are obtained, the ray tracing simulation can be performed using these matrices as inputs. The source $\Sigma$ was located at the center of the cavity and a circular detector of radius $\delta=a / 2$ was put at an arbitrary position: $\Delta_{x}=1.17 \mathrm{~m} \hat{x}$ and $\Delta_{y}=0.58 \mathrm{~m} \hat{y}$, relative to the center of the cavity. The number of rays used for the calculation was $N=100000$. Short runs (with $N=20000$ ) were performed in order to check the influence of the source/receiver position on the averaged EDIR quantities. Air absorption as a function of frequency was incorporated into the model using standard data from tables. ${ }^{8}$ Rays were traced until their energy decayed $60 \mathrm{~dB}$.

Figure 4 illustrates the averaged EDIR for the cavity. The RED [Fig. 4(a)] exhibits resonances for certain frequency bands (the energy remains within the cavity for long periods of time). The longest and widest resonance corresponds to the total band gap of the lattice (0.865 to $1.25 \mathrm{kHz}$ ), while other shorter resonances occur for narrower frequency bands within the regions where the lattice has partial band gaps.

The Angular Energy Density is displayed in Fig. 4(b). A feature that is immediately apparent is the localization of the energy over certain angular regions for the resonances that corresponds to the partial band gaps. This corresponds to a highly anisotropic intensity field within the cavity. By contrast, for the full band gap frequency band an almost isotropic energy density is found. These results are independent of the source/receiver position.

\section{B. Hexagonal lattice}

Next, we calculate the impulse response of the same cavity as before but using hexagonal lattice sonic crystals as walls, with lattice parameter $a=0.3 \mathrm{~m}$. For the hexagonal lattice, the scattering properties of each wall $(\hat{x}$ and $\hat{y}$ direction) are different due to the lack of symmetry of the structure relative to a right angle rotation. Then, it is needed to perform two MST calculations with different position vectors for the cylinders. For the wall oriented along the $\hat{x}$ direction, the position vectors are given by: $R_{l \hat{x}}=l \sqrt{3} / 2 a \hat{X}$ $+a / 2[$ lis even $] \hat{Y}$ and $R_{\alpha \hat{x}}=\alpha a \hat{Y}$. While for the wall oriented along the $\hat{y}$ direction the vectors are: $R_{l \hat{y}}=l a / 2 \hat{X}+$ $\sqrt{3} / 2 a[$ l is even $] \hat{Y}$ and $R_{\alpha \hat{y}}=\alpha \sqrt{3} a \hat{Y}$.

All the simulation parameters are the same as in the square lattice with the only exception of the source position that is displaced $L_{x} / 4$ in the positive $\hat{x}$ direction.

Figures 5(a) and 5(b) illustrate the Reflectivity Index matrices $(R)$ for the walls oriented along the $\hat{x}$ and $\hat{y}$ directions, respectively. In order to easily compare the results with the ones obtained for the square lattice we keep the ratio $\lambda / a$ constant by setting the maximum frequency of the simulation in $2.5 \mathrm{kHz}$. In this case, there are no full band gaps, and all the total reflection regions occur for certain incidence angle ranges. These regions are apparently different comparing the $R$ matrices for the two orientations. A more intricate pattern is observed for the wall oriented along the $\hat{y}$ direction, especially for frequencies above $1 \mathrm{kHz}$. This is reasonable since this wall is not oriented along any primitive crystal axis.
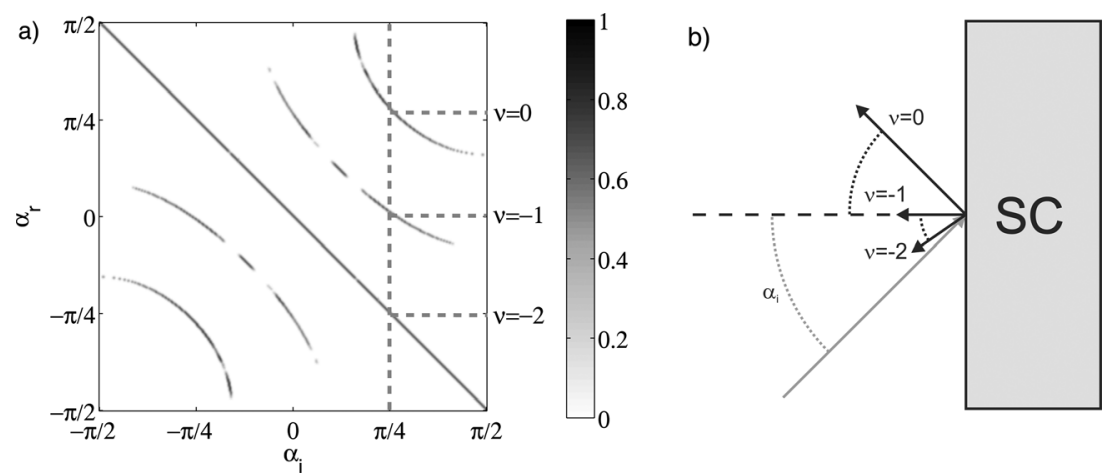

FIG. 3. Scattered angle probability density $\mathrm{S}$ for a square lattice sonic crystal infinite slab obtained using MST. (a) The scattered angle probability density $S\left(f, \alpha_{i}, \alpha_{r}\right)$ gives the probability for a ray of incidence angle $\alpha_{i}$ and associated frequency $f$ of being reflected with an angle $\alpha_{r}$. We display a matrix $S$ corresponding to a frequency of $2.5 \mathrm{kHz}$. (b) Schematic representation of a ray impinging a sonic crystal infinite slab with incidence angle $\alpha_{i}=\pi / 4$ and the reflected rays with the corresponding Bragg orders: specular $(\nu=0)$ and higher order reflections $(\nu=-1,-2)$. As a reference, this incidence angle is traced on the $S$ matrix, showing the intersection with the Bragg reflections. 

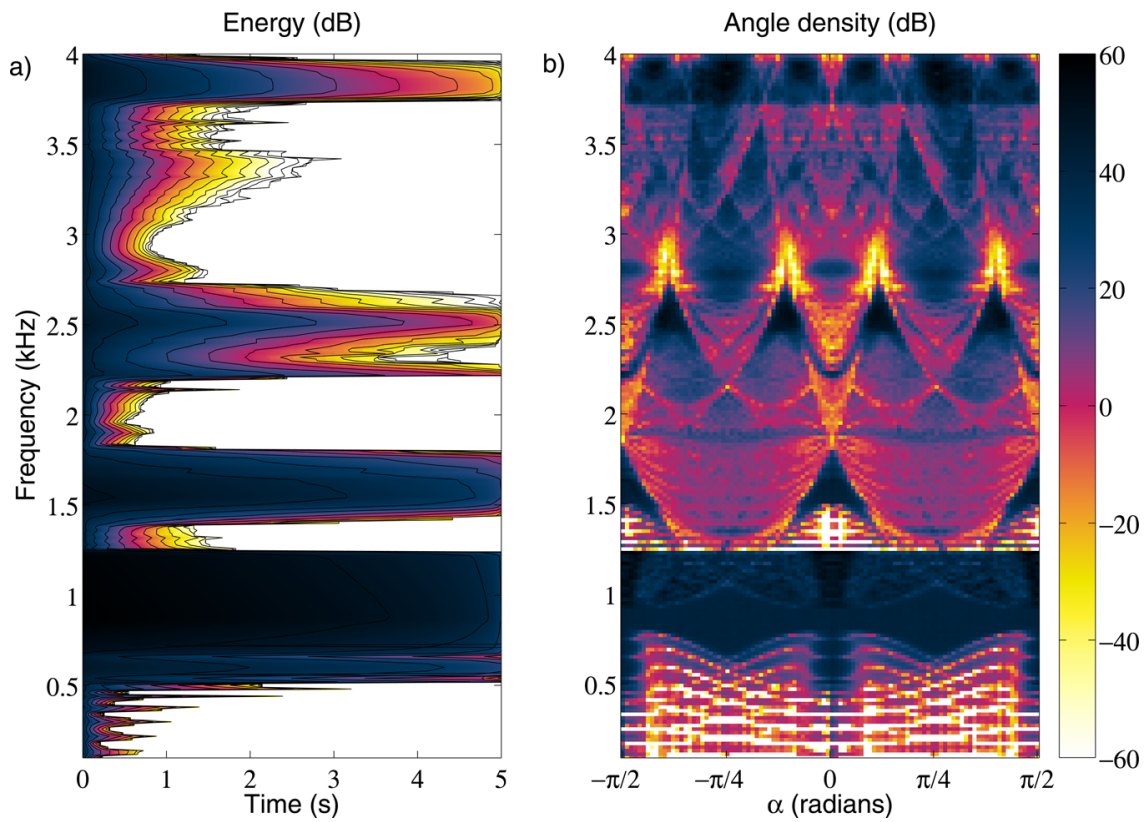

FIG. 4. (Color online) Averaged EDIR quantities for a cavity with square lattice sonic crystals walls with the same parameters as Fig. 2. (a) Remaining Energy Density $[\operatorname{RED}(f, t)$, see Eq. (33)], which shows the temporal evolution of the energy remaining in the cavity at the receiver position as a function of frequency. (b) Angular Energy Density $[A E D(f, t)$, see Eq. (34)], which shows the time-averaged angular distribution for the energy of the rays arriving at the receiver position (in $\mathrm{dB} / \mathrm{Hz}$ ) as a function of frequency.

As a final case, we introduce a perturbation on the hexagonal lattice by displacing the even rows a distance $a / 4$ in the positive $\hat{x}$ direction. After the displacement, the position vectors of the cylinders for the wall oriented along the $\hat{x}$ direction are given by: $R_{l \hat{x}}=l \sqrt{3} / 2 a \hat{X}+3 a / 4[$ l is even $] \hat{Y}$ and $R_{\alpha \hat{x}}=\alpha a \hat{Y}$. And for the wall oriented along the $\hat{y}$ direction the vectors are: $R_{l \hat{y}}=a / 2(l+[$ l is even $] / 2) \hat{X}+\sqrt{3} / 2 a$ [l is even $] \hat{Y}$ and $R_{\alpha \hat{y}}=\alpha \sqrt{3} a \hat{Y}$. The ray tracing calculation parameters remains the same as in the unperturbed hexagonal array.
Figures 5(c) and 5(d) depict the Reflectivity Index matrices $(R)$ for the walls of the perturbed hexagonal lattice, oriented along the $\hat{x}$ and $\hat{y}$ directions, respectively. By comparing these matrices with the corresponding matrices for the unperturbed case, it can be noticed that the main effect of the perturbation is to reduce the size of the regions of total reflection above $1 \mathrm{kHz}$. Thus, as the lattice becomes less symmetric, the complexity of $R$ increases.

Figure 6 shows the RED of the cavity for the case of hexagonal [Fig. 6(a)] and perturbed hexagonal [Fig. 6(b)] a)

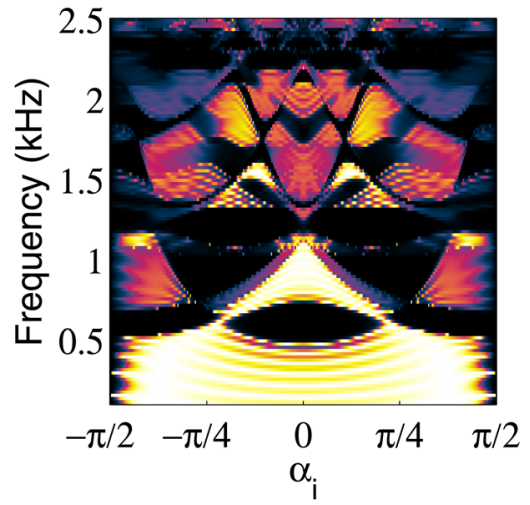

c)

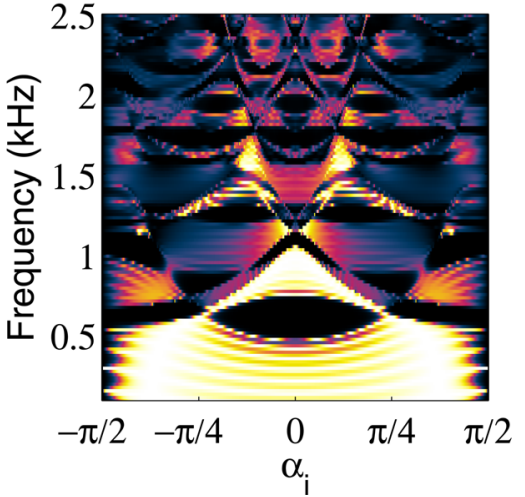

b)

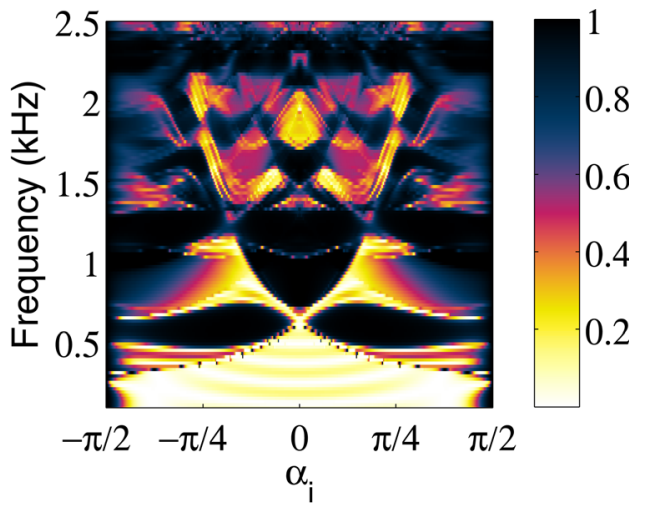

d)

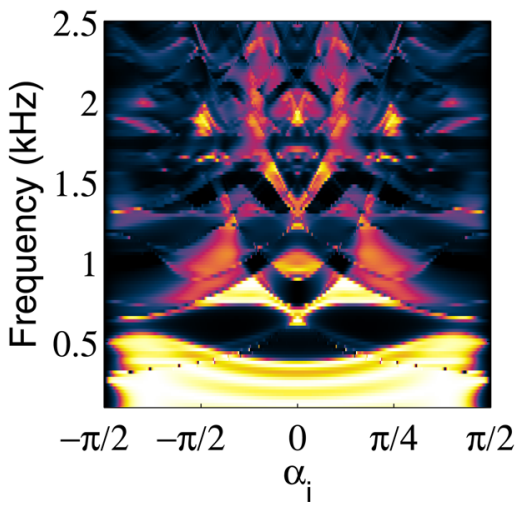

FIG. 5. (Color online) Reflectivity index matrices $R$ for (a and b) a hexagonal lattice and (c and d) a displaced hexagonal lattice sonic crystal infinite slabs obtained using MST. The lattice constant is $a=0.3$ $\mathrm{m}$ and the radius of the cylinders $\rho$ $=0.08 \mathrm{~m}$. The displaced lattice was obtained by shifting the even rows a distance of $a / 4$ in the $\hat{x}$ direction. Left plots [Figs. 5(a) and 5(c)] corresponds to the walls oriented in the $\hat{x}$ direction and right plots [Figs. 5(b) and $5(\mathrm{~d})]$ corresponds to the perpendicular walls. 

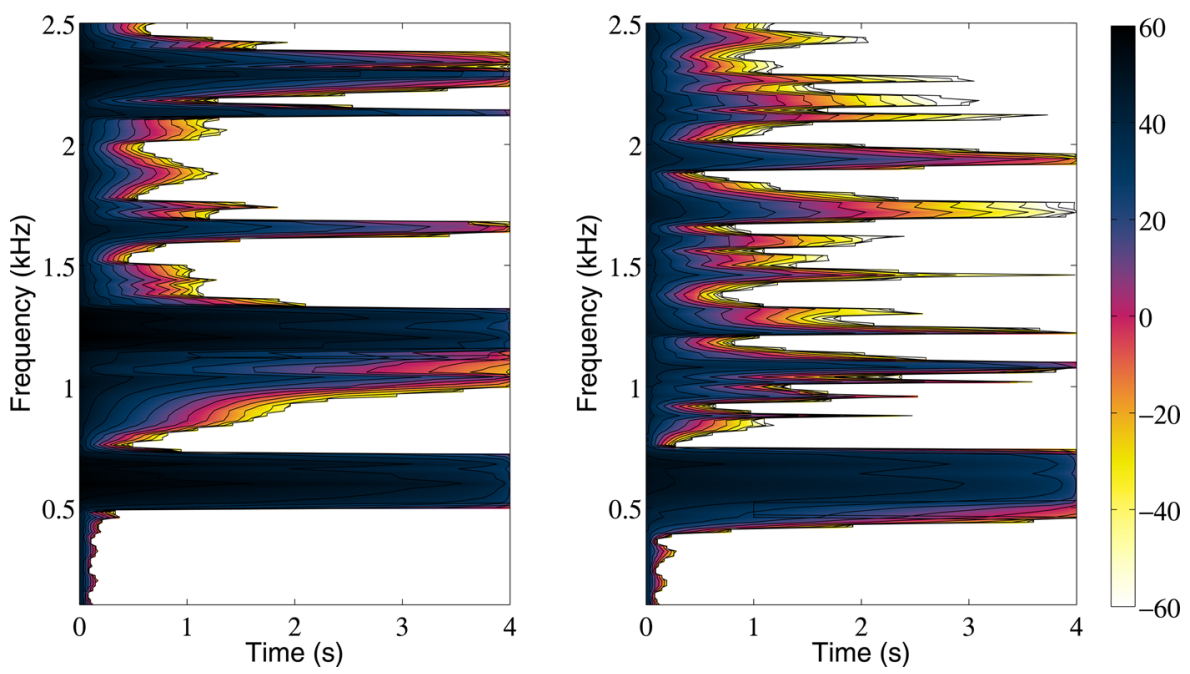

FIG. 6. (Color online) Time evolution of the remaining energy $R E D(f, t)$ inside the cavity with (a) hexagonal lattice sonic crystal walls, and (b) displaced hexagonal lattice sonic crystal walls, using the same parameter values as Fig. 5.

sonic crystal walls. As with the square sonic crystal walls, the strongest resonance is observed for the first band gap (between 0.5 and $0.7 \mathrm{kHz}$ ), and shorter resonances occur for subsequent partial band gaps. The main effect of the perturbation is to spread and reduce the duration of the strongest resonance, and to break down the higher resonances into smaller peaks, generating a more spectrally distributed energetic impulse response.

\section{DISCUSSION}

In this section, we analyze the results obtained with the hybrid method and derive approximations from geometrical acoustics for the distribution of total reflection regions on the reflectivity index matrix and the structure of the resonances inside the cavity.

\section{A. Total reflection condition}

We begin by considering the reflectivity index matrix $R\left(f, \alpha_{i}\right)$, defined by Eq. (30) and depicted in Fig. 2 (Fig. 5) for the square (hexagonal) lattice. A common feature that emerges from these plots is their structure of arches, corresponding to the consecutive Bragg orders. As a reference, a curve for the beginning of the first order $(k a / 2 \pi)\left(\sin \alpha_{i}-1\right)$ $=-1$ is displayed in Fig. 2(a). The subsequent orders are obtained from this curve as integer multiples and reflections along the normal-incidence axis. This structure is a direct consequence of the periodicity of the cylinders along the tangent direction of the slab that acts as a diffraction grid, and can be obtained even with a single row. As more rows are added, total reflection areas $(R=1)$ develop at both sides of the curves limiting the Bragg orders. Also, fringes corresponding to the Fabry-Pérot interference of the slab, appear flanking the total reflection areas. ${ }^{18}$ Note that for the hexagonal lattice cleaved along the $\hat{y}$ direction [Fig. 5(b)] the number of Bragg orders is higher, and the spacing between them is smaller by a factor of $1 / \sqrt{3}$, because the periodicity of the surface is larger by a factor of $\sqrt{3}$.

Since the sonic crystal is two-dimensional (or, equivalently the cylinders are infinite) it is expected that for the forbidden bands (band gap) the energy of the incident waves cannot propagate inside the sonic crystal, being reflected back and forming the total reflection regions in the $R$ matrix. This explains the total reflection band for all angles between 0.865 and $1.25 \mathrm{kHz}$ for the square lattice, corresponding to the total band gap of the SC.

For the partial band gaps of the SC total reflection occurs only for certain ranges of incidence angles. The prediction of these bands requires more elaborated calculations. A fairly good approximation can be obtained using the PWE method and the Snell's law. Even when both approaches are valid only for a semi-infinite medium, the results obtained are pretty similar to those gained from MST using a sufficient number of rows, at least for the first frequency bands. We proceeded as follows. From the dispersion relation obtained using PWE we derive the group velocity as the gradient of the frequency surfaces in the reciprocal space and the effective group refraction index $\left(n_{\text {eff }}\right)$ as a function of the wave vector $\left(\mathbf{k}^{\prime}\right)$ inside the SC. Then, for a fixed frequency and using the Snell's law with $n_{\text {eff }}\left(\mathbf{k}^{\prime}\right)$, we derived all the possible incident wave vectors $(\mathbf{k})$ corresponding to the allowed values of $\mathbf{k}^{\prime}$ for that frequency. The obtained angles of incidence corresponds to modes that can propagate inside the $\mathrm{SC}$, and for which total reflection do not occur. In other words, total reflection regions are formed by those wave vectors $\mathbf{k}$ which cannot excite any propagation mode inside the SC. For SC slabs with a finite number of rows these regions are thinner because even when propagation modes are absent, the energy can cross the slab due to tunneling effects.

In Fig. 7(a) we display the results for the boundary of the first band with total reflection for the $R$ matrix of a semiinfinite square lattice calculated using PWE (dashed line), and 16 infinite rows using MST (solid line). The difference between the two curves for oblique incidence angles is due to the finite width of the wall. As more rows are added, the boundary obtained using MST approaches the curve calculated using PWE. A feature that is immediately apparent for both curves is the lack of symmetry with respect to an incidence angle of $\pi / 4$, even when this is a symmetry axis of the crystal. This can be easily explained using geometrical acoustics. For this particular frequency band, the forbidden propagation directions are close to the normal and tangent 
a)

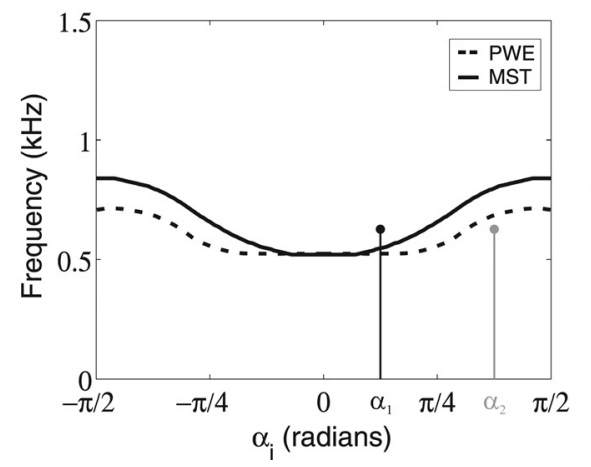

b)

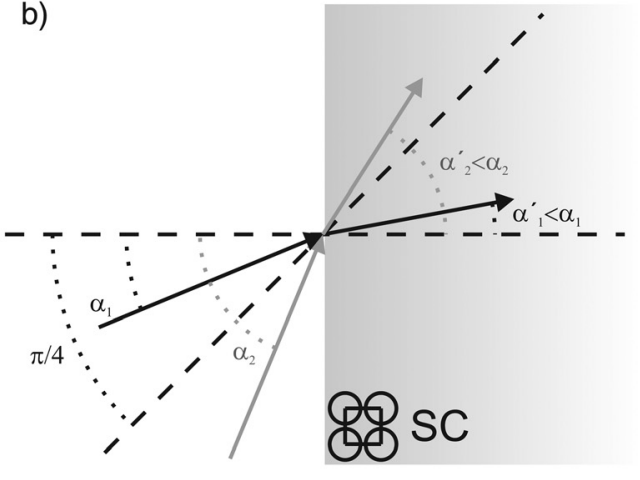

FIG. 7. Prediction of total reflection zones for a semi-infinite medium using the PWE method and the Snell's law. (a) Boundary of the first band with total reflection for the R matrix of a semi-infinite square lattice calculated using PWE (dashed line), and 16 infinite rows using MST (solid line). (b) Schematic representation of two rays impinging a semi-infinite square lattice SC with angles $\alpha_{1}$ and $\alpha_{2}$, that are symmetric with respect to the $\pi / 4$ direction. The ray with incidence angle $\alpha_{1}$ is refracted towards the forbidden normal direction (and cannot propagate inside the SC), while the other ray is refracted towards the allowed direction $\pi / 4$.

incidence angles [vector pointing to $X$ in the primitive cell, see Fig. 2(b)], and the allowed propagation directions are close to $\pm \pi / 4$ incidence angles (vector pointing to $M$ in the primitive cell). The lack of symmetry arises because $n_{\text {eff }}$ is always greater than one, then rays with small incidence angles are refracted towards the forbidden normal direction, while very oblique rays are refracted away from the forbidden tangent direction. This is illustrated in Fig. 7(b) using two rays with angles of incidence $\alpha_{1}$ and $\alpha_{2}$, that are symmetric with respect to the $\pi / 4$ direction and have an associated frequency such that the first (second) ray corresponds to a forbidden (allowed) $\mathbf{k}^{\prime}$ propagation vector inside the SC.

\section{B. Resonances of the cavity}

Next we turn on analyzing the resonances observed in the Remaining Energy Densities displayed in Figs. 4 and 6. These resonances corresponds to rays that persist within the cavity for several seconds, undergoing up to a few hundreds of reflections. Only rays with combinations of frequency and incidence angles that give a reflectivity index very close to one will survive after such a number of reflections. Moreover, since the cavity is rectangular, we require that total reflection occurs for certain incidence angles and their complementary angles as well, because rays can bounce between walls oriented along the $\hat{x}$ and $\hat{y}$ directions.

We consider three possibilities for these long-term rays: (a) rays with associated frequencies for which total reflection occurs for every angle of incidence, (b) rays undergoing specular reflections with associated frequencies for which total reflection occurs for complementary angles, and (c) rays reflecting in one or more higher Bragg orders with associated frequencies for which total reflection occurs for the Bragg angles and their complementary ones. Here, for the sake of clarity, we discuss in detail only the two firsts cases, but the same arguments can be extended to higher Bragg orders.

The first case occurs for the full band gap regions, where trivially all rays are trapped inside the cavity, loosing energy only due to air absorption. This explains the first, longer, and broad resonance in Fig. 4.
For the second case, we compute the probability of remaining with a non-vanishing energy value, after many wall reflections, as a function of the angle of incidence of the ray, using the information contained in the $R$ matrix and geometrical considerations. Since we are only dealing with specular reflections, a ray impinging a front wall with angle $\alpha_{0}$, will undergo $n_{1}$ reflections with angle of incidence $\alpha_{0}$ on the front and rear walls, and $n_{2}$ reflections with angle of incidence $\pi / 2-\alpha_{0}$ on the sidewalls, where $n_{1}$ depends on the initial angle and the aspect ratio of the room, and $n_{1}+n_{2}$ is equal to the total number of reflections. The ray energy after such a number of reflections is multiplied by a factor $R\left(f, \alpha_{0}\right)^{n_{1}} R\left(f, \pi / 2-\alpha_{0}\right)^{n_{2}}$. As the number of reflections grows, this factor does not vanish only if $R\left(f, \alpha_{0}\right)$ and $R\left(f, \pi / 2-\alpha_{0}\right)$ are very close to one.

In Fig. 8(b) we display the matrix obtained from of this calculation. In order to compare this result with the probability obtained using the hybrid method [see Fig. 8(a)], we also multiply the matrix by the probability for the initial angle $\alpha_{0}$, that depends on the source position (as the source is well apart from the walls the probability vanishes toward the tangent directions $\pm \pi / 2$ ). This explains why the resonances corresponding to partial band gaps occur only for certain narrow frequency bands, and why there is always a highly anisotropic intensity field associated to that resonance [see Fig. 4(b)].

These resonances are of a completely different nature than the usual resonances (modes) for enclosures, since they are not wave related and they just depend on the geometry of the sonic crystal and the cavity. Furthermore, they produce highly anisotropic intensity fields, where the sound only propagates along certain restricted directions. This last feature is absent in conventional rooms (with the possible exception of having concave walls, but even in this case the direction and frequency are fixed). Furthermore, what is most interesting is that the resonances can be modified by changing the internal geometry of the walls, as was illustrated with the perturbed hexagonal lattice.

We close by discussing some possible applications for the system studied here. In principle, a physical realization 
a)

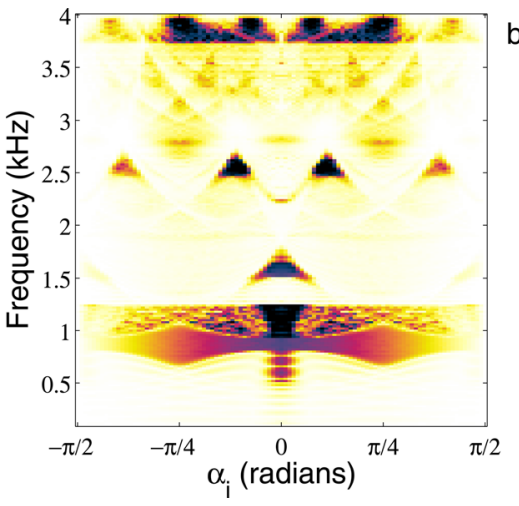

b)

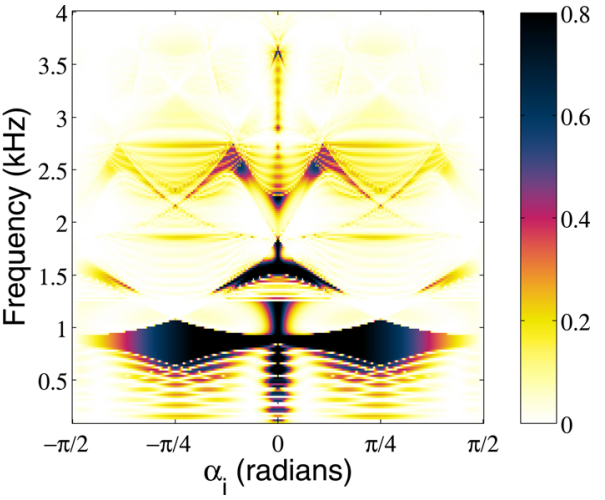

FIG. 8. (Color online) Comparison between the density of incidence angles $\alpha_{i}$ on the walls of the cavity inside a square lattice SC (a) calculated by using the hybrid method, and (b) estimated for rays undergoing specular reflections by using the reflectivity index matrix and geometrical data. of this two-dimensional "Sonic Crystal Room" could be possible, provided that long enough cylinders and cylindrical sound sources are used. The unconventional characteristics of such a room could be incorporated, for example, into venues for contemporary music ${ }^{19}$ (where sound spatialization is a major concern). A minimal version of the SC walls, probably with fewer rows and correspondingly with weaker and more subtle resonances, can also be used to modify the acoustics of existing rooms. The main advantage of the SC walls when compared to conventional walls is the possibility of adjusting their resonances by changing their internal geometry. Therefore, it is also possible to think of an "acoustic coating" for walls made by SCs that can be adjusted to equalize the room for a particular purpose. In this way, rooms with variable acoustics can be obtained with minimal effort.

\section{CONCLUSIONS}

We have proposed a successful hybrid method, combining MST and geometric acoustics, for calculating the energetic directional impulse response of a large cavity inside a two dimensional sonic crystal made by rigid cylinders in air. A reflectivity index matrix and a scattering probability density were derived from MST calculations. These magnitudes were analyzed for three different sonic crystal geometries: square lattice, hexagonal lattice, and a perturbed hexagonal lattice. The structure observed in the reflectivity index matrix was related to the Bragg modes and, for the first frequency band, predicted by a simple calculation using PWE and the Snell's law. Afterward, these matrices were used for a modified ray-tracing algorithm that incorporates Bragg diffraction. The temporal and directional energy densities obtained with this method displayed strong resonances for certain frequency bands and, in some cases, a highly anisotropic intensity field. A simple calculation was used to show that these resonances correspond to the existence of regions of total reflection for certain frequency bands and complementary angles on the reflectivity index matrix.

\section{ACKNOWLEDGMENTS}

We acknowledge Daniel Torrent for useful discussions. This work was partially funded by CONICET, ANPCyT, and UNQ.
${ }^{1}$ J. V. Sanchez-Perez, D. Caballero, R. Martinez-Sala, C. Rubio, J. Sánchez-Dehesa, F. Meseguer, J. Llinares, and F. Gálvez, "Sound attenuation by a two-dimensional array of rigid cylinders," Phys. Rev. Lett. 80, 5325-5328 (1998).

${ }^{2}$ L. Feng, X.-P. Liu, M.-H. Lu, Y.-B. Chen, Y.-F. Chen, Y.-W. Mao, J. Zi, Y.-Y. Zhu, S.-N. Zhu, and N.-B. Ming, "Acoustic backward-wave negative refractions in the second band of a sonic crystal," Phys. Rev. Lett. 96, 014301 (2006).

${ }^{3}$ M.-H. Lu, C. Zhang, L. Feng, J. Zhao, Y.-F. Chen, Y.-W. Mao, J. Zi, Y.Y. Zhu, S.-N. Zhu, and N.-B. Ming, "Negative birefraction of acoustic waves in a sonic crystal," Nat. Mater. 6, 744-748 (2007).

${ }^{4} \mathrm{~B}$. Gupta and Z. Ye, "Theoretical analysis of the focusing of acoustic waves by two-dimensional sonic crystals," Phys. Rev. E 67, 036603 (2003).

${ }^{5}$ L. Sanchis, F. Cervera, J. Sánchez-Dehesa, J. V. Sanchez-Perez, C. Rubio, and R. Martinez-Sala, "Reflectance properties of two-dimensional sonic band-gap crystals," J. Acoust. Soc. Am. 109, 2598-2605 (2001).

${ }^{6}$ Y. Wang, K. Deng, S. Xu, C. Qiu, H. Yang, and Z. Liu, “Applications of antireflection coatings in sonic crystal-based acoustic devices," Phys. Lett. A 375, 1348-1351 (2011).

${ }^{7}$ M.-H. Lu, L. Feng, X. J. Liu, Y. Cheng, Y.-Y. Zhu, Y. Mao, and J. Zi, "Experimental observation of both negative and positive phase velocities in a two-dimensional sonic crystal," Phys. Lett. A 366, 523-527 (2007).

${ }^{8}$ A. C. Gade, Acoustics in Halls for Speech and Music, in Springer Handbook of Acoustics, edited by T. D. Rossing (Springer, New York, 2007), pp. 301-349.

${ }^{9}$ M. Kushwaha, "Stop-bands for periodic metallic rods: Sculptures that can filter the noise," Appl. Phys. Lett. 70, 3218-3220 (1997).

${ }^{10}$ Y.-Y. Chen and Z. Ye, "Theoretical analysis of acoustic stop bands in two-dimensional periodic scattering arrays," Phys. Rev. E 64, 036616 (2001).

${ }^{11}$ J. O. Vasseur, P. A Deymier, A. Khelif, Ph. Lambin, B. Djafari-Rouhani, A. Akjouj, L. Dobrzynski, N. Fettouhi, and J. Zemmouri, "Phononic crystal with low filling fraction and absolute acoustic band gap in the audible frequency range: A theoretical and experimental study," Phys. Rev. E. 65, 056608 (2001).

${ }^{12}$ H. Kuttruff, Room Acoustics (Spon Press, London, 2000), pp. 101-126.

${ }^{13} \mathrm{~V}$. Twersky, "Multiple scattering of radiation by an arbitrary planar configuration of parallel cylinders and by two parallel cylinders," J. Appl. Phys. 23, 407-414 (1952).

${ }^{14} \mathrm{P}$. Waterman, "New formulation of acoustic scattering," J. Acoust. Soc. Am. 45, 1417-1429 (1969).

${ }^{15}$ J. Sánchez-Dehesa, V. M. Garcia-Chocano, D. Torrent, F. Cervera, S. Cabrera, and F. Simon, "Noise control by sonic crystal barriers made of recycled materials," J. Acoust. Soc. Am. 129, 1173-1183 (2011).

${ }^{16} \mathrm{D}$. Torrent, Towards the full control of sound with sonic crystals and acoustic metamaterials, Ph.D. thesis, Universidad Politécnica de Valencia (2008).

${ }^{17}$ M. Schroeder, "New method of measuring reverberation time," J. Acoust. Soc. Am. 37, 409-412 (1965).

${ }^{18}$ L. Sanchis, A. Håkanson, F. Cervera, J. Sánchez-Dehesa, "Acoustic interferometers based on two-dimensional arrays of rigid cylinders in air," Phys. Rev. B 67, 035422 (2003).

${ }^{19}$ J. Harrison and S. Wilson, "Sound and space: New approaches to multichannel music and audio," Organised Sound 15, 183-184 (2010). 